\title{
USING PHYSICAL LOGIC GATES TO TEACH DIGITAL LOGIC TO NOVICE COMPUTING STUDENTS
}

\author{
Wee Lum Tan and Sven Venema \\ School of ICT, Griffith University, Brisbane, Queensland, Australia
}

\begin{abstract}
One of the challenges that commencing university students in computing degree programs face is the difficulty in engaging with the abstract and complicated theories in the computing discipline. In particular, it is hard for beginner computer architecture students to visualise the link between the theory of digital logic and the behaviour of the digital circuitry. Studies have shown that improving disciplinary engagement can lead to lower student attrition rates. We aim to increase student engagement in an introductory computer architecture course by employing oversized physical logic gates in the form of logic blocks in several digital logic experiments. Using these digital logic blocks students will be able to physically interact with the circuitry and observe the outcomes of their solutions directly. Student survey results indicate that using the physical logic blocks was helpful to beginner computing students in gaining a better understanding of digital logic and digital circuits. The use of physical logic blocks was also helpful in transitioning to a more abstract digital logic drawing environment later in the course for building more advanced digital circuits.
\end{abstract}

\section{KEYWORDS}

Digital Logic Gates, Digital Circuits, Logicblocks, Logisim

\section{INTRODUCTION}

Commencing university students face a multitude of challenges (Baik, Naylor, \& Arkoudis, 2015). A successful transition to university can be facilitated by supporting students across a number of common issues as outlined in Lizzio's Five Senses of Success model (Lizzio, 2006; Lizzio \& Wilson, 2010). Supporting students at the start of their academic career is particularly important in computing fields where student attrition rates are typically higher than other Science Technology Engineering and Mathematics (STEM) fields (US Department of Education, 2013). One of the issues identified in (Lizzio, 2006) relates to the level of disciplinary engagement. Resilience can be developed by students through improved disciplinary engagement, and this can have a positive flow-on effect on student attrition. In this paper we examine the impact of using a physical computing environment on student engagement with digital logic theory in a first-trimester introductory computer architecture course.

Based on previous student feedback we believe that one of the challenges facing students in engaging with the digital logic component of the course relates to the abstract nature of the theory. Students learning about digital logic in this course will be exposed to a number of digital logic gates (AND, OR, NOT, NAND, NOR, XOR) and how these logic gates are combined to create more complex digital logic circuits such as a simple addition circuit. A significant barrier to student understanding of the workings of the individual logic gates is that it is not possible to see inside a digital logic gate to understand how it works. Instead, the student must develop a linkage between the behaviour of the circuit and its name and symbol. Students then use these symbols to chain together multiple logic gates to build more complex circuitry. In past iterations of the course, logic gates and circuits of this type could either be drawn on paper or using a digital logic drawing program that can simulate electrical current flowing through the circuit and test the circuit. The benefit of the digital logic drawing program approach is that the student can experiment and test their circuit. However, past student feedback has indicated frustration with the drawing program which is considered too abstract and complicated when students have only just learnt the digital logic concept. Frustration at this early part of the course can lead to disengagement with the topic. 
There is growing evidence that using some form of physical computing modules in addition to the more traditional approaches is beneficial to the learning experience of beginner computing students (Richard, 2010; Rubio, Romero-Zaliz, Manoso, \& de Madrid, 2014; Maia, da Silva, de S. Rosa, Queiroz-Neto, $\&$ de Lucena, 2009). In a similar vein, we believe that the use of physical logic blocks may also be beneficial when these students learn about digital logic. In support of this belief, a study in a digital design course using a programmable logic device (Radu \& Cole, 2008) has indicated that using these types of hands-on devices has had a positive impact on the development of discipline specific skills and student intention of continuing with their program of study. Having access to a physical unit can give less abstract feedback to the student on the behaviour of the logic gate and circuit. This can help to reduce some of the uncertainty of the beginning student.

The focus of this paper is improving disciplinary engagement in the teaching of digital logic in a computing degree. We aim to achieve this by using popular low cost physical digital logic blocks (LogicBlocks, n.d.) that can be connected to each other to build moderately complex circuits. Using these digital logic blocks students will be able to physically observe the outcomes of their solutions to problems, rather than as static written text on a page or as a graphical representation on a screen. They will be able to interact with the circuitry and observe the outcome of their solution directly. This hands-on approach will facilitate experimentation, allow students to evaluate the effectiveness of the solution, and aid in the development of alternate solutions.

We have incorporated several experiments using the digital logic blocks into the laboratory classes at the start of the course to reinforce the digital logic theory introduced in lectures. The use of digital logic blocks is intended as a scaffold for the more abstract digital logic drawing program (Logisim n.d.) used in subsequent laboratories and in the main course assignment. The results indicate that the LogicBlocks activity was generally successful in helping the novice students gain a better understanding of digital logic and digital circuits, with some limitations related to the complexity of circuits that can be built due to the size of the LogicBlocks kit.

The remainder of this paper is organised as follows. In Section 2, we describe the digital logic teaching tools used in the course as well as the experiments using the digital logic blocks. In Section 3 we give an overview of the methodology. We present and discuss the student feedback on the experiments in Section 4 and offer our conclusions in Section 5.

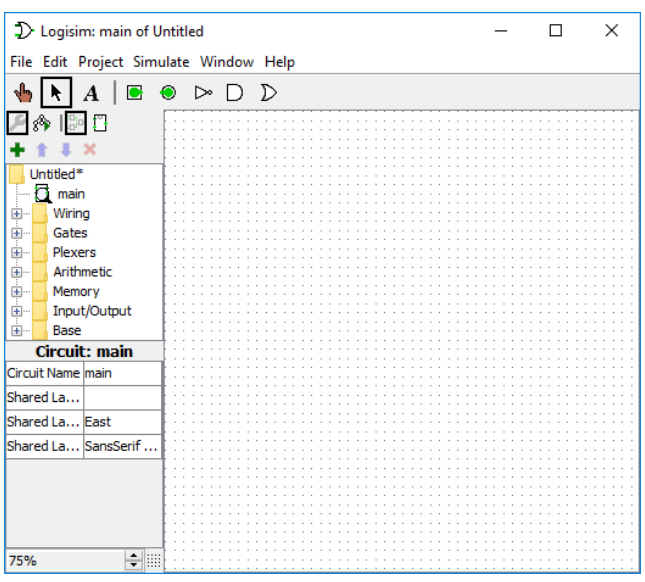

Figure 1a. Logisim Simulator

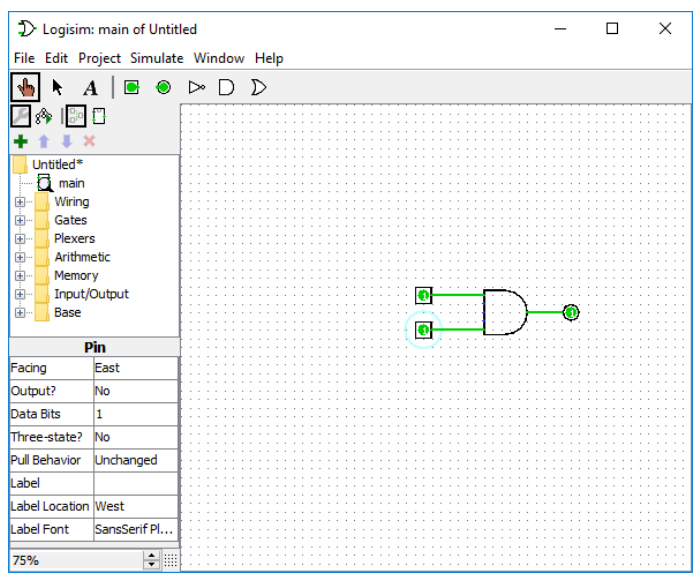

Figure 1b. AND Gate in Logisim

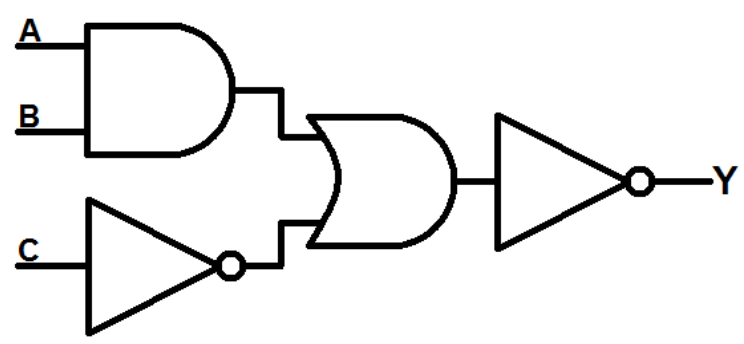

Figure 2. Combinatorial Digital Logic Experiment on Logisim 


\section{DIGITAL LOGIC TEACHING TOOLS}

In the introductory computing course on computer architecture, students are introduced to the underlying digital components and logic that define a computer. The basic logic gates (AND, OR, NOT, NAND, NOR, $\mathrm{XOR}$ ) and their corresponding truth tables are presented and then used to construct more advanced digital circuits such as combinatorial and sequential logic circuits. These topics are first taught in lectures, and then practised and reinforced during exercises in laboratory classes.

In previous offerings of the course, students have used the Logisim simulator tool (Logisim, n.d.) for designing and simulating digital logic circuits. The laboratory exercises use Logisim to build and evaluate basic logic gates (see Figure 1) as well as more advanced circuits (see Figure 2). Indicative student comments related to the digital logic component focus on the lack of "hands-on practical experience with hardware", with suggestions of incorporating "more practical stuff with gates - using gates \& how they work with binary" and "more kinaesthetic learning resources when covering logic gates would have been great since the concept was quite difficult for many people". These comments have been sourced from formal universitywide anonymous end-of-trimester course evaluations in previous offerings of the course.

To address the students' need for practical hands-on exercises with logic gates, we have introduced the SparkFun LogicBlocks electronic kit (LogicBlocks, n.d.) into the course. The LogicBlocks electronic kit (see Figure 3) allows the students to play and experiment with physical logic gates, thereby enabling them to see how the output of the digital circuit reacts to the inputs. We have incorporated a set of laboratory exercises using the LogicBlocks electronic kits to scaffold the students' learning and understanding of the theory and practical aspects of digital logic gates. The teaching of digital logic is structured so that students first work on these LogicBlocks laboratory exercises (see Sections 2.1, 2.2, 2.3). After having been exposed to LogicBlocks, students continue their study of digital logic in the following weeks with the Logisim simulator tool and its associated laboratory exercises. Logisim allows students to create more complex and advanced circuits that would be challenging to create with LogicBlocks due to the limitation on the number of available components in the physical kits. In Sections 2.1, 2.2, and 2.3 we discuss the LogicBlocks experiments in more detail.

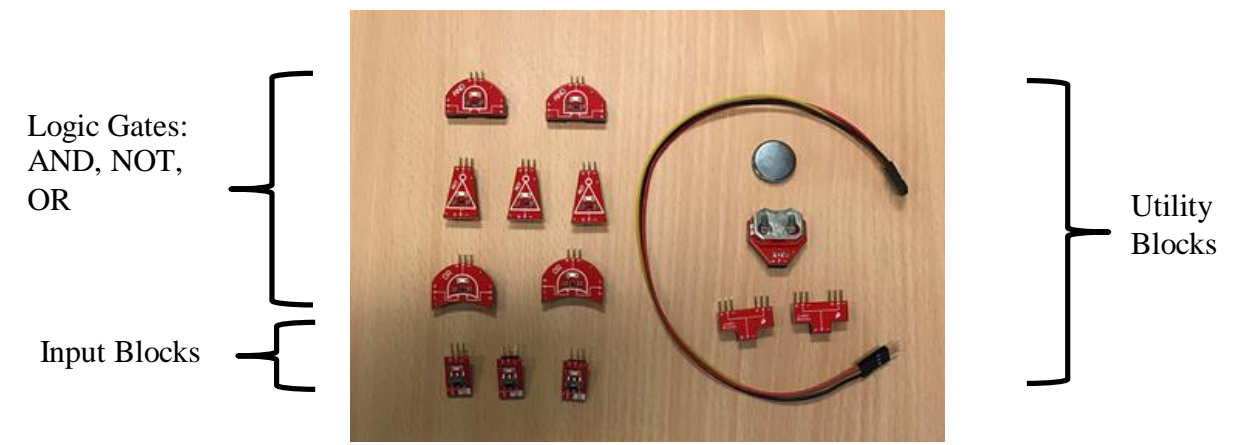

Figure 3. LogicBlocks Kit: Logic Gates, Input Blocks, and Utility Blocks

\subsection{Basic Digital Logic Gate Experiments}

In this laboratory exercise, students are asked to construct an AND gate logic circuit shown in Figure 4, experiment with different combinations of input signals $(0=\mathrm{OFF}, 1=\mathrm{ON})$ and fill up the truth table for an AND logic gate. A truth table is a tabular representation of the possible input states and their corresponding output state. Students are also asked to repeat the same exercise for different logic gates (OR, NOT, NAND, NOR). This laboratory exercise is designed to reinforce the students' understanding of the basic logic gate functions and characteristics. 


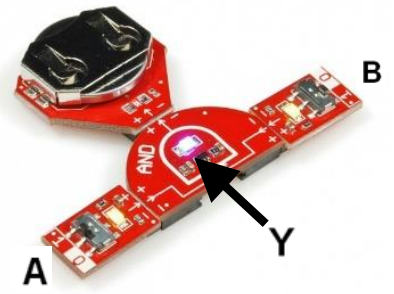

\begin{tabular}{|c|c|c|}
\hline Input A & Input B & Output $\mathbf{Y}$ \\
\hline 0 & 0 & 0 \\
\hline 0 & 1 & 0 \\
\hline 1 & 0 & 0 \\
\hline 1 & 1 & 1 \\
\hline
\end{tabular}

Figure 4. AND Logic Gate and its Truth Table

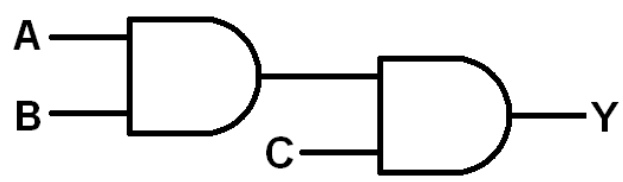

Figure 5. Combinatorial Digital Logic Experiment

\subsection{Combinatorial Digital Logic Circuit Experiment}

In this laboratory exercise, students are asked to construct a more advanced combinatorial digital logic circuit (see Figure 5) with the LogicBlocks kit. Students are asked to try out all possible input combinations for $\mathrm{A}, \mathrm{B}$ and $\mathrm{C}$, and construct the truth table for the circuit. This exercise trains the students to follow the progression of the input signals to the output signal $\mathrm{Y}$, as well as help the students take the first step in understanding how more complicated circuits are essentially constructed from simple basic logic gates.

\subsection{Oscillator Digital Logic Circuit Experiment}

In this laboratory exercise, students are asked to construct the oscillator circuit shown in Figure 6, and explain what they see when the battery power is plugged in. Similar to the exercise in Section 2.2, this exercise requires the students to follow the progression of the input signals to the output signal, and additionally gives the students an opportunity to see for themselves how a sequential logic circuit works with the output signal feedback into the circuit input. One benefit of using LogicBlocks is that this type of oscillating circuit can easily be created, whereas in the Logisim simulator this may not be able to be implemented correctly if the simulation is not completely accurate.

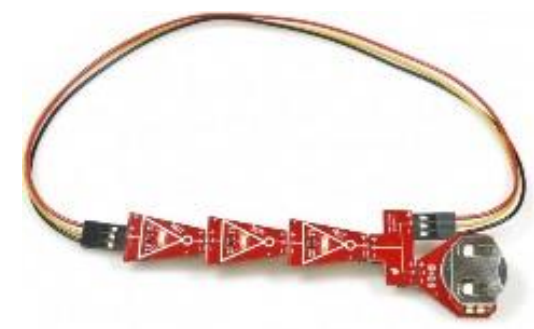

Figure 6. Oscillator Digital Logic Experiment

\section{METHODOLOGY}

In the introductory computing course on computer architecture students attend two hours of lectures and 2 hours of laboratories during each of the 12 teaching weeks. The 377 enrolled students are distributed across two campus offerings with identical content, assessment, and delivery modes. The LogicBlocks experiments discussed in Sections 2.1, 2.2 and 2.3 were completed by students at both campuses in their week 4 laboratory class after having completed three hours of digital logic lectures covering the basic logic gates. After completing the LogicBlocks activity, students used the Logisim simulator to develop more advanced digital logic circuits from week 5 onwards.

Students were surveyed twice to better gain an understanding of their experience with both LogicBlocks and Logisim. Survey 1 was held in the laboratory class in week 5, one week after the week 4 LogicBlocks 
experiments outlined in Sections 2.1, 2.2, and 2.3. Survey 2 was held in week 6 after students had used the Logisim simulator to develop the digital logic circuit in Figures 1 and 2 for their week 5 activity.

The questions for Survey 1 were developed to gain an understanding of how effective students perceived the LogicBlocks activities to be in reinforcing their understanding of the basic digital logic gates. For Survey 2 , the questions were aimed at understanding if the LogicBlocks activities were successful in preparing students for the use of the Logisim simulator. The survey questions are shown in Table 1 and Table 2. For Survey 1, Questions 1, 2, and 3 in Table 1 ask students to grade their experience on a 5-point Likert-style scale ranging from Strongly Agree through to Strongly Disagree. In Table 1, Questions 4 and 5 are open-ended questions where students can give a more detailed textual response. For Survey 2, Questions 1, 2, 3 , and 4 in Table 2 ask students to grade their experience on a 5-point Likert-style scale ranging from Strongly Agree through to Strongly Disagree with the option for Q2 and Q4 to give an open-ended textual response if they chose a negative response. Student responses were anonymous and there were 140 and 187 responses for Survey 1 and Survey 2, respectively.

Table 1. Survey 1 Questions on the LogicBlocks Experiments

\begin{tabular}{|c|l|}
\hline Question & Description \\
\hline Q1 & Do you have any prior knowledge of digital logic and digital circuits before taking this course? \\
\hline Q2 & After attending and revising Lecture 3, I am confident in my knowledge of digital logic and digital circuits \\
\hline Q3 & $\begin{array}{l}\text { The hands-on LogicBlocks activity in Workshop 4 helped me gain a better understanding of digital logic } \\
\text { and digital circuits }\end{array}$ \\
\hline Q4 & What did you like the most about the LogicBlocks activity? \\
\hline Q5 & What did you like the least about the LogicBlocks activity? \\
\hline
\end{tabular}

Table 2. Survey 2 Questions on the LogicBlocks and Logisim Experiments

\begin{tabular}{|c|l|}
\hline Question & Description \\
\hline Q1 & $\begin{array}{l}\text { The hands-on LogicBlocks activity in Workshop 4 helped me gain a good understanding of digital logic } \\
\text { and digital circuits }\end{array}$ \\
\hline Q2 & $\begin{array}{l}\text { Completing the hands-on LogicBlocks activity in Workshop 4 was a useful preparation for working with } \\
\text { the Logisim simulator in Workshop 5 }\end{array}$ \\
\hline Q3 & $\begin{array}{l}\text { The Logisim simulator activity in Workshop 5 was a useful way to build on the understanding of logic } \\
\text { circuits that was developed using LogicBlocks in Workshop 4 }\end{array}$ \\
\hline Q4 & $\begin{array}{l}\text { The hands-on LogicBlocks activity was more helpful to me in gaining a good understanding of digital } \\
\text { logic and digital circuits, compared to the Logisim simulator }\end{array}$ \\
\hline
\end{tabular}

\section{RESULTS AND DISCUSSION}

In Survey 1,99 out of 140 students ( $71 \%$ of respondents) indicated that they did not have any prior knowledge of digital logic and digital circuits before taking the course (Figure 7). From these 99 students, $44 \%$ answered that they were confident (Agree and Strongly Agree) in their knowledge of digital logic learnt in the lecture before undertaking the LogicBlocks activity (Q2 in Figure 8). In contrast, only 9\% of the 99 students felt that they were not confident (Disagree and Strongly Disagree) in their knowledge even after the lecture but before the LogicBlocks activity. 47\% of the 99 students gave a Neutral response to Q2 indicating uncertainty about their level of confidence. After the hands-on LogicBlocks activity, 68\% of the 99 respondents who said No in Q1 agreed in Q3 that the LogicBlocks activity was helpful in them gaining a better understanding of digital logic. Only 6\% felt that the activity didn't help them (Q3 in Figure 8).

Looking at the responses in more detail, of the 9 out of 99 respondents ( 1 respondent $\sim=1 \%$ of this study group) who Disagreed with Q2 in Survey 1, 4 of these respondents now agreed in Q3 that LogicBlocks helped them to better understand digital logic. 3 people responded with Neutral for Q3, and 2 disagreed that LogicBlocks helped them to better understand digital logic. The 2 negative respondents indicated that they felt the content was moving too fast and that they would like a lecture specifically on LogicBlocks to prepare for the LogicBlocks activity.

From the 44 respondents who were confident in their knowledge of digital logic in Q2, 1 person responded with Strongly Disagree to Q3 with the comment that the LogicBlocks were flimsy. It is possible that their response regarding the utility of LogicBlocks was negative partly due to build quality issues with 
the kits. Another 4 of these students who agreed to Q2, responded with Neutral to Q3. Their comments indicated that they felt that the activity was rushed and that they felt constrained in what they could build due to the small number of gates available in the kits.

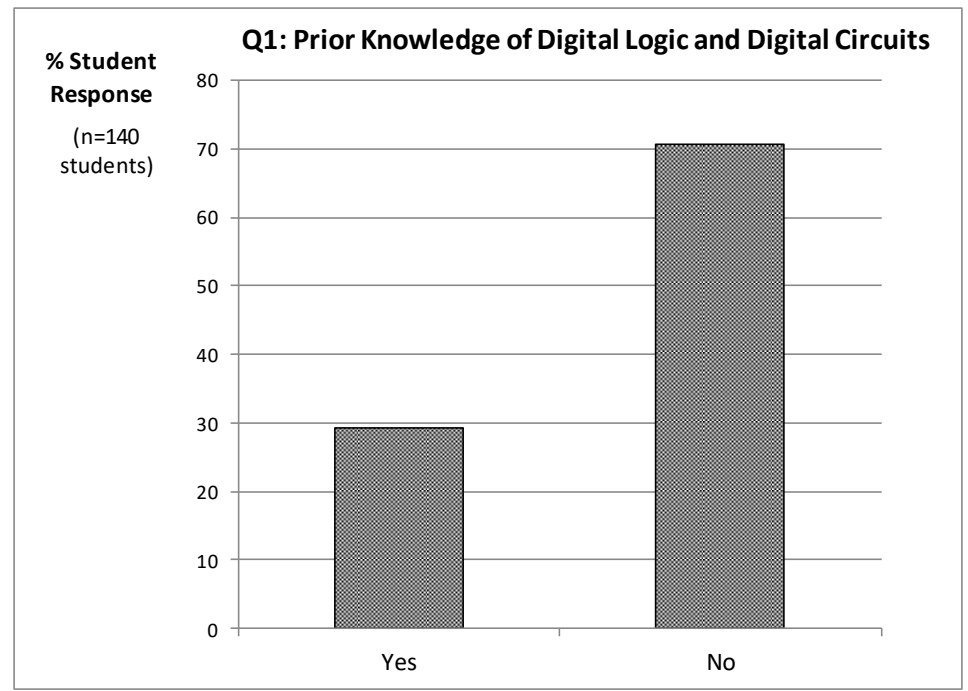

Figure 7. Student Responses to Survey1:Q1 on Prior Knowledge of Digital Logic and Digital Circuits

Of the 46 respondents who answered with Neutral to Q2, 3 of these respondents disagreed with Q3 that the LogicBlocks activity helped them to gain a better understanding of digital logic. Their comments indicated that they did not like the activity, felt it was pointless and that it "did not help my understanding at all'. These 3 students were in the minority ( $3 \%$ of the 99 respondents).

Survey 1 responses also revealed that 41 students ( $29 \%$ of respondents) indicated that they have had some prior knowledge of digital logic and digital circuits before taking the course (Figure 7). From these 41 students, $80 \%$ of them answered that they were confident in their knowledge of digital logic before undertaking the LogicBlocks activity (Q2 in Figure 9) and 78\% of the students agreed that the LogicBlocks activity helped them gain a better understanding of digital logic and digital circuits (Q3 in Figure 9). This shows that even those students who already had prior knowledge of digital logic before the course, felt that they benefited from the hands-on LogicBlocks activity.

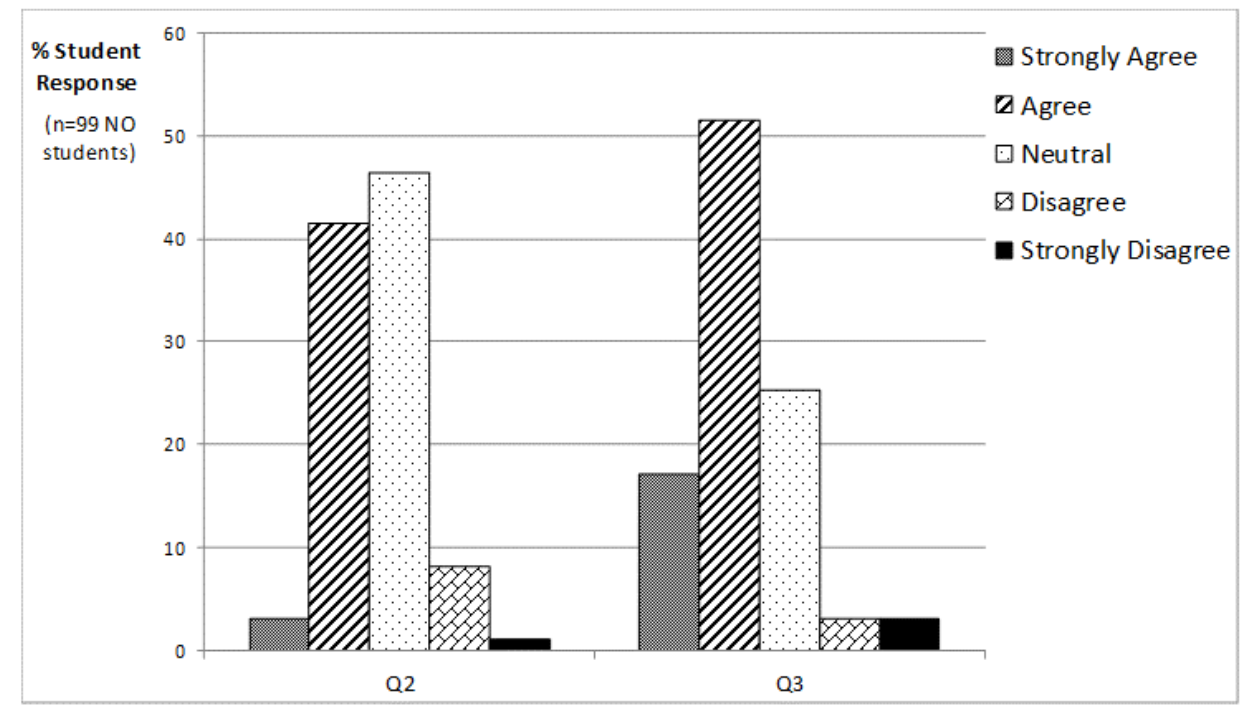

Figure 8. Responses to Survey1:Q2 and Q3 from Students without Prior Knowledge of Digital Logic 


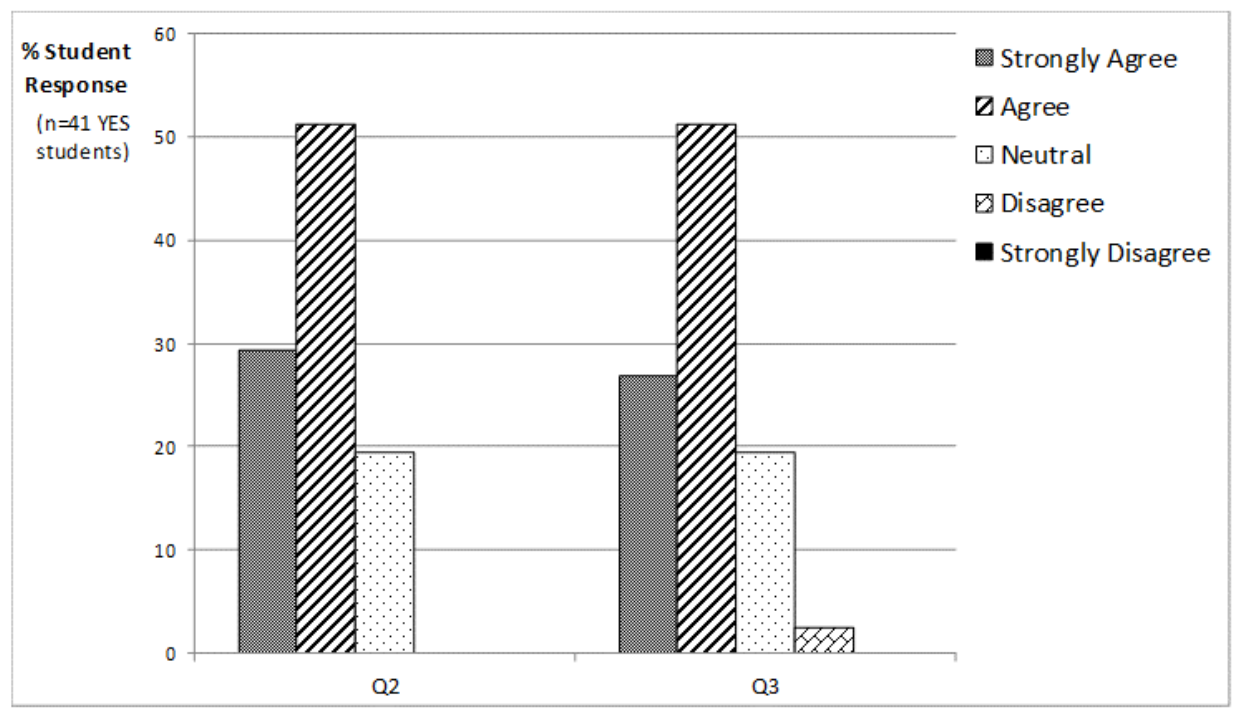

Figure 9. Responses to Survey1:Q2 and Q3 from Students with Prior Knowledge of Digital Logic

Overall when combining the responses from both sets of students (those with and without prior knowledge of digital logic and digital circuits), we found that $71 \%$ of the students agreed that the LogicBlocks activity was helpful to them while only $5 \%$ of the students disagreed. Student comments were very positive, with many of them stating that they found it useful being able to physically work with the logic gates and circuits, and visually see how the circuits work and respond to different input combinations. Some sample student comments: "It gave us hands on, practical experience with circuits and logic gates" and "The Logic Block gate kit used in one of the computer labs was exceedingly useful in promoting understanding of how logic gates work and interactive together to form a single circuit". These results indicate that the LogicBlocks activity was generally successful in helping the novice students gain a better understanding of digital logic and digital circuits.

For Survey 2 in Q1, students were again asked whether they felt the LogicBlocks activity was helpful to them in gaining a better understanding of digital logic and digital circuits. The responses reveal that $71 \%$ of the students agreed (Q1 in Figure 10), which is a similar percentage of students who agreed in Q3 of Survey 1. In addition, $66 \%$ of the students agreed that the LogicBlocks activity was a useful preparation for working with the Logisim simulator, while only a small number (7.5\%) of students disagreed (Q2 in Figure 10). These results are further proof that the LogicBlocks activity was beneficial to the majority of students.

The Survey 2 responses also show that $79 \%$ of the students agreed that the Logisim simulator activity was a useful way to build on their understanding of logic circuits that was developed with the LogicBlocks activity (Q3 in Figure 10). Finally, when comparing LogicBlocks and the Logisim simulator in Q4, 47.5\% of the students felt that LogicBlocks was more helpful to them in gaining an understanding of digital logic and digital circuits, while 19.2\% of the students disagreed (Q4 in Figure 10). The reasonably high percentage of disagreeing students can be at least partially attributed to the fact that the LogicBlocks kit was fairly restricted in the types of complex digital circuits that can be constructed, compared with what is possible with the Logisim simulator. This view is supported by some open-ended comments such as "with logicblocks we were limited in what we could do whilst we had all of Logisim at our disposal." and "the simulator provides more opportunities to make your own circuits, thus gain a more comprehensive understanding without limitations of the Logic-Block activity". This could in part be addressed in future offerings of the course by expanding the size of the LogicBlocks kits available to each student and developing activities and experiments for more complex circuitry. 


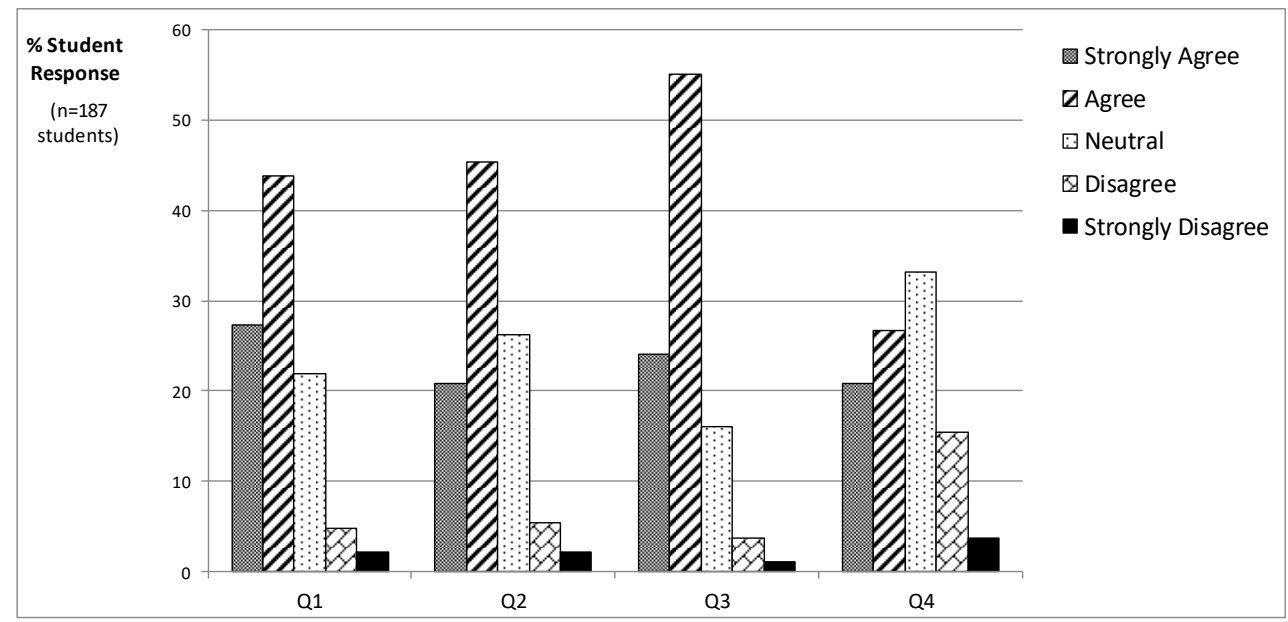

Figure 10. Student Responses to Survey2:Q1 to Q4 on LogicBlocks and Logisim

\section{CONCLUSIONS}

We have carried out several digital logic experiments using physical logic gates (LogicBlocks) in an introductory Computer Architecture course. The aim of these experiments is to gain an understanding of the impact of using a physical computing environment on student engagement with digital logic. Student feedback indicates that using the physical logic blocks was helpful in gaining a better understanding of digital logic and digital circuits and was helpful in transitioning to a more abstract digital logic drawing environment (Logisim simulator). Scaffolding student learning of digital logic is important for developing student understanding of how a computer works.

For future work, we plan to expand the size of the LogicBlocks kits available to each student and develop more complex activities and experiments using a larger number of logic blocks to address student feedback regarding the lack of complexity available to them with the current LogicBlocks kit.

\section{REFERENCES}

Baik, C., Naylor, R., \& Arkoudis, S. (2015). The First Year Experience in Australian Universities: Findings from Two Decades, 1994 - 2014. Melbourne Centre for the Study of Higher Education (CSHE), University of Melbourne.

Lizzio, A. (2006). Designing an Orientation and Transition Strategy for Commencing Students: A Conceptual Summary of Research and Practice. First Year Experience Project. Griffith University.

Lizzio, A. \& Wilson, K. (2010). Strengthening Commencing Students' Sense of Purpose: Integrating Theory and Practice. Proceedings of the 13th Pacific Rim First Year in Higher Education Conference.

LogicBlocks (n.d.). Retrieved from https://www.sparkfun.com/products/11006

Logisim. (n.d.). Retrieved from http://www.cburch.com/logisim/

Maia, L.D.O., da Silva, V.J., de S. Rosa, v.E.V., Queiroz-Neto, J.P. \& de Lucena, V.F. (2009). An Experience to use Robotics to Improve Computer Science Learning. Proceedings of the $39^{\text {th }}$ IEEE Frontiers in Education Conference.

Radu, M. E. \& Cole, C. (2008). Enabling creative thinking in digital design education. The Annual Conference for the European Society for Engineering Education, (SEFI), Aalborg, Denmark.

Richard, G. T. (2010). Employing Physical Computing in Education: How Teachers and Students Utilized Physical Computing to Develop Embodied and Tangible Learning Objects. The International Journal of Technology, Knowledge and Society, vol. 4, pp. 93-102.

Rubio, M. A., Romero-Zaliz, R., Manoso, C. \& de Madrid, A.P. (2014). Enhancing an Introductory Programming Course with Physical Computing Modules. Proceedings of the 2014 IEEE Frontiers in Education Conference (FIE).

US Department of Education. (2013). STEM Attrition: College Students' Paths Into and Out of STEM Fields Statistical Analysis Report, NCES 2014-00. Retrieved from https://nces.ed.gov/pubs2014/2014001rev.pdf 